\title{
Pandemia de influenza: la respuesta de México
}

\author{
Pablo Kuri-Morales, MC, M en C,(1) Miguel Betancourt-Cravioto, MC, MSc, \\ Oscar Velázquez-Monroy, MC, MISS, EEA, ${ }^{(2)}$ Carlos Alvarez-Lucas, CD, MISS, EEA, ${ }^{(3)}$ \\ Roberto Tapia-Conyer, MC, MSP, MSc, Dr.Sc. ${ }^{(4)}$
}

\section{Kuri-Morales P, Betancourt-Cravioto M, Velázquez-Monroy O, Alvarez-Lucas C,Tapia-Conyer R. Pandemia de influenza: la respuesta de México. Salud Publica Mex 2006;48:72-79.}

\section{Resumen}

En 1992 apareció en el sureste asiático un nuevo tipo de virus de la influenza, el cual ha ocasionado hasta la fecha más de 120 casos y un poco más de 60 defunciones en humanos en Camboya, Vietnam, Indonesia y Tailandia. Esta situación es considerada por los expertos como la probable génesis de una nueva pandemia de influenza, lo que podría traer graves consecuencias para la salud de la población, así como para la economía y el comercio mundial. Por lo anterior, la Organización Mundial de la Salud (OMS) ha instado a los países miembros a desarrollar planes de preparación y respuesta para hacer frente a esta eventualidad. En el marco del Comité Nacional para la Seguridad en Salud, México ha diseñado el Plan Nacional de Preparación y Respuesta ante una Pandemia de Influenza con objeto de proteger a la población mediante acciones efectivas y oportunas. El Plan utiliza una escala de riesgo y define cinco líneas de acción: Coordinación,Vigilancia Epidemiológica, Atención Médica, Difusión y Movilización Social,y Reserva Estratégica. Si bien es imposible predecir cuándo se presentará la próxima pandemia y su impacto, es fundamental que las autoridades de salud nacionales, estatales y locales establezcan los mecanismos para poner en marcha los componentes del Plan en forma oportuna y garantizar con ello la salud de la población en caso de influenza pandémica.

Palabras clave: pandemia de influenza; influenza aviar;preparación y respuesta; emergencia en salud pública; México

\author{
Kuri-Morales P, Betancourt-Cravioto M, \\ Velázquez-Monroy O,Alvarez-Lucas C,Tapia-Conyer R. \\ Influenza pandemic: Mexico's response. \\ Salud Publica Mex 2006;48:72-79.
}

\section{Abstract}

In 1992, a new type of influenza virus appeared in Southeast Asia. This new strain has caused to date, more than 120 cases and over 60 deaths in Cambodia, Vietnam, Indonesia and Thailand. This situation is seen by the experts as the possible genesis of a new influenza pandemic with the corresponding negative effects on the health of the population, international commerce and world economy. In order to face the coming challenge, the World Health Organization (WHO) has asked member countries to develop national preparedness and response plans for an influenza pandemic. Within the framework of the National Committee for Health Security, Mexico has developed a National Preparedness and Response Plan for an Influenza Pandemic with the aim of protecting the health of the population with timely and effective measures. The Plan is based on a risk scale and five lines of action: Coordination, Epidemiological Surveillance, Medical Care, Risk Communication and Strategic Stockpile. It is currently impossible to predict when the next pandemic will start or what will be its impact. Nevertheless, it is fundamental that national and regional health authorities establish measures for protecting the health of the population in case this emergency occurs.

Key words: influenza pandemic; avian influenza; preparedness and response; public health emergencies

(I) Dirección General de Epidemiología. Secretaría de Salud. México, D.F., México.

(2) Centro Nacional de Vigilancia Epidemiológica y Control de Enfermedades. Secretaría de Salud. México, D.F., México.

(3) Dirección General de Programas Preventivos. Secretaría de Salud. México, D.F., México.

(4) Subsecretaría de Prevención y Promoción de la Salud. Secretaría de Salud. México, D.F., México.

Solicitud de sobretiros: M en C Pablo Kuri Morales. Dirección General de Epidemiología. Secretaría de Salud.

Francisco de P. Miranda 177, $4^{\circ}$ piso, Lomas de Plateros, Delegación Alvaro Obregón, 01480 México, D.F.

Correo electrónico: pkuri@dgepi.salud.gob.mx 
E 1 término pandemia de influenza se refiere a la ocurrencia masiva de casos, con una elevada tasa de infección y mortalidad, ocasionada por la aparición de un nuevo subtipo de virus A, contra el cual la población no tiene inmunidad natural. ${ }^{1}$

En general, las pandemias de influenza condicionan un incremento significativo en la demanda de consultas médicas, altas tasas de hospitalización y de muerte. Tienen un efecto importante en la economía y en el bienestar social como consecuencia del ausentismo laboral y de la limitación del flujo de personas y de mercancías entre países y regiones, lo que puede originar una grave disrupción de los servicios básicos y de salud. ${ }^{2}$

Desde luego, es difícil predecir cuándo ocurrirá una pandemia, aunque sí sabemos que se presentan con cierta regularidad. En el siglo XX, por ejemplo, ocurrieron tres pandemias: en 1918, 1957 y $1968 .^{2}$

En la última década, varias cepas del virus de la influenza aviar han cruzado los límites entre aves y humanos y causado la muerte en algunos enfermos: el H7N7 en Holanda, el H9N2 en Hong Kong y el H7N2 en Nueva York. 3,4 Sin embargo, el subtipo H5N1 de 1992 es el que ha puesto al mundo más cerca de una nueva pandemia desde que ocurrió en 1968. Ello se debe principalmente a la habilidad del virus de causar enfermedad en hombres y mujeres sin la necesidad de contar con genes de virus humanos, además de su intensa sintomatología y elevada letalidad. ${ }^{4}$

El virus de influenza aviar de alta patogenicidad A H5N1 ya es enzoótico en aves del sureste asiático, en donde las autoridades no han podido contener el brote en animales, por lo que continúa la acumulación de casos de la enfermedad en humanos. Actualmente, la enfermedad aviar se ha extendido hacia el oeste a través de las rutas migratorias, afectando a aves silvestres y de granja de países del centro de Asia y del este y sur de Europa (cuadro I). ${ }^{5}$ No obstante hay quienes argumentan que, con la magnitud del brote en aves, si el H5N1 fuera la causa de la pandemia, ésta ya se hubiera presentado. ${ }^{3}$

Hoy en día dos de las tres condiciones generales para el inicio de una pandemia se han cumplido: la emergencia de un nuevo virus de influenza y la habilidad de dicho virus para replicarse en humanos causando enfermedad grave. El cumplimiento de la tercera condición, esto es, que el virus obtenga la propiedad de transmisión eficiente humano-humano, podría condicionar el inicio de la próxima pandemia de influenza. Por lo mismo, una de las principales limitantes para las autoridades sanitarias es precisamente predecir si el virus adquirirá esta propiedad o no, y cuándo ocurrirá, 6 o cuál será el grado de virulencia del

\section{Cuadro I \\ Casos acumulados hasta enero 9 de 2006 DE INFLUENZA AVIAR POR A(H5NI) EN HUMANOS}

\begin{tabular}{lcc} 
País & Casos & Defunciones \\
Indonesia & 16 & II \\
\hline Tailandia & 22 & 14 \\
\hline Camboya & 4 & 4 \\
\hline Vietnam & 90 & 39 \\
\hline China & 7 & 3 \\
\hline Turquía & 4 & 2 \\
\hline Total & 143 & 73
\end{tabular}

Fuente: Organización Mundial de la Salud, 8 de enero de 2006

nuevo virus y su impacto en morbilidad y mortalidad entre los seres humanos. ${ }^{1}$

Sin duda, es factible que ocurra esta tercera condición dado el elevado riesgo de mutación o reacomodo genético del virus, lo que podría generar que éste sea de alta transmisibilidad. ${ }^{5}$

Un escenario ideal sería contar con una vacuna específica que permita la protección, pero como no conocemos realmente al virus, la prioridad está en identificar intervenciones efectivas de salud pública encaminadas a detener la pandemia en sus etapas iniciales, tales como el uso profiláctico de antivirales o bien contar con estrategias para reducir las tasas de infección entre la población (distanciamiento social) y la difusión entre la población de las medidas de higiene básicas para evitar el contagio de la enfermedad (uso de cubrebocas, lavado de manos, buena alimentación, etc.). La efectividad de tales políticas dependerá de qué tan rápido pueden ser diagnosticados los casos clínicos y la velocidad de distribución de los antivirales. ${ }^{5}$

En cualquier caso, las acciones de prevención servirán para proteger a la población mundial, sea cual sea la cepa que cause la próxima pandemia, o que ésta ocurra por el paso de una cepa aviar al hombre o por cambios antigénicos importantes en un patógeno humano conocido. ${ }^{3}$

Por lo anterior, la OMS ha instado a los gobiernos a desarrollar planes multidisciplinarios de prevención y control para una eventual pandemia de influenza. ${ }^{7}$

La respuesta a una pandemia de influenza debe prepararse con todo cuidado y todas las opciones disponibles deben ser consideradas y evaluadas en cuanto a su utilidad y costo/beneficio. Todos los sistemas de apoyo deben utilizarse para informar oportunamente a la población, a la comunidad médica y a los responsables de la respuesta para aplicar oportuna- 
mente las medidas necesarias y así mitigar los efectos de la pandemia. Se debe evitar el pánico y mantener el orden público, situaciones que pudieran generarse de no tener perfectamente establecidos los mecanismos de respuesta correspondientes. ${ }^{1}$

De acuerdo con lo sugerido por la OMS, los objetivos de la planeación en el plano global para una pandemia de influenza se orientan a: ${ }^{8}$

1. Reconocer de manera oportuna la emergencia de cepas virales de potencial pandémico y monitoreo de su evolución.

2. Establecer mecanismos que permitan identificar el inicio de la pandemia de influenza.

3. Reducir la morbilidad, mortalidad e ingresos hospitalarios por la enfermedad.

4. Desarrollar la capacidad para atender un elevado número de individuos enfermos graves en la comunidad y en hospitales.

5. Asegurar que los servicios esenciales se mantengan funcionando para reducir al máximo la disrupción de la vida diaria.

6. Brindar información adecuada, oportuna y actualizada al personal de salud, población general, medios de comunicación y todos aquellos grupos que lo requieran.

Todos ellos contribuirán sin duda al objetivo común de protección global contra el riesgo de una pandemia, incidiendo a la vez en la reducción del impacto de la misma en las tasas de enfermedad y muerte.

\section{Desarrollo del Plan Nacional de Prepara- ción y Respuesta ante una Pandemia de Influenza de México}

Desde 2003, en México se institucionalizó el concepto de seguridad en salud, como uno de los componentes centrales de la seguridad nacional. A través de la creación del Comité Nacional para la Seguridad en Salud (CNSS), el país cuenta con un órgano colegiado del más alto nivel encabezado por la Secretaría de Salud, con la responsabilidad de analizar, definir, dar seguimiento y evaluar las políticas nacionales en materia de seguridad en salud, lo que le ha conferido el carácter de una política de estado. ${ }^{9}$

Las acciones del CNSS se sustentan en cinco ejes fundamentales: emergencias hospitalarias, brotes de enfermedades infecciosas, exposición a agentes químicos, desastres naturales y bioterrorismo.

En este contexto se conformó un grupo de trabajo con el objetivo de desarrollar el Plan Nacional de Preparación y Respuesta ante una Pandemia de Influenza. El grupo se encuentra conformado por todas las instituciones públicas federales que integran al Comité, las cuales participan de manera activa en el diseño y actualización permanente de los lineamientos técnico-normativos del Plan, desde una perspectiva integral y multidisciplinaria y atendiendo a las competencias de cada institución participante (cuadro II).

El objetivo del Plan es proteger a la población mexicana mediante la instrumentación oportuna de acciones coordinadas para hacer frente a las necesidades de atención con la finalidad de reducir el impacto de un evento de esa naturaleza en el país. ${ }^{10}$

Con todos estos propósitos se ha buscado que las estrategias incorporadas en el Plan se basen en la evidencia científica internacional, y las experiencias nacionales, privilegiando, ante todo, que surjan del consenso de todos los actores relevantes involucrados en el país.

Las acciones de preparación y respuesta del Plan se encuentran organizadas en líneas de acción, las cuales adquieren características diferentes de acuerdo con el nivel de riesgo para la población y una escala desarrollada específicamente para nuestro país, que, a su vez, se basa en una escala formulada por la OMS (cuadro III).

Para fines de planeación, en esta escala se asume, siguiendo los escenarios epidemiológicos actuales, que la pandemia iniciará en otro continente, muy probablemente en Asia. Sin embargo, la escala es flexible y permite pasar de una fase a otra sin tener que recorrer todas las fases precedentes. Debido a que las fases reflejan riesgo cada vez mayor para la presencia de casos en territorio mexicano, si la pandemia iniciara en Europa, el riesgo inicial sería mayor que si lo hiciera en Asia, debido al intenso flujo de personas y mercancías entre los países europeos y México.

Por otro lado, en la fase prepandémica en México, se plantean las acciones de vigilancia epidemiológica

\section{Cuadro II}

Comité Nacional para la Seguridad en Salud. INSTITUCIONES INTEGRANTES DEL GRUPO DE TRABAJO DE PANDEMIA DE INFLUENZA

- Secretaría de Salud

- Secretaría de Gobernación (Coordinación General de Protección Civil)

- Secretaría de Agricultura, Ganaderia, Desarrollo Rural, Pesca y Alimentación

- Secretaría de la Defensa Nacional

- Secretaría de Marina

- Instituto Mexicano del Seguro Social (regimen ordinario y Oportunidades)

- Instituto de Seguridad y Servicios Sociales para los Trabajadores del Estado

- Sistema Nacional para el Desarrollo Integral de la Familia

- Petróleos Mexicanos 


\begin{tabular}{|c|c|c|c|c|c|c|c|c|}
\hline \multicolumn{9}{|c|}{$\begin{array}{c}\text { Cuadro III } \\
\text { EsCALA DE RIESGO }\end{array}$} \\
\hline \multicolumn{3}{|c|}{ Prepandemia } & \multicolumn{3}{|c|}{ Alerta pandémica } & \multicolumn{3}{|c|}{ Pandemia } \\
\hline $\begin{array}{l}\text { Fases } \\
\text { OMS }\end{array}$ & $\begin{array}{l}\text { No hay nuevos virus } \\
\text { de influenza en seres } \\
\text { humanos }\end{array}$ & $\begin{array}{l}\text { Nuevo subtipo de } \\
\text { virus en animales con } \\
\text { riesgo para humanos }\end{array}$ & $\begin{array}{l}\text { Casos en humanos sin } \\
\text { transmisión humano- } \\
\text { humano }\end{array}$ & $\begin{array}{l}\text { Brotes m } \\
\text { humanos, } \\
\text { misión lim }\end{array}$ & $\begin{array}{l}4 \\
\text { enores en } \\
\text { con trans- } \\
\text { itada }\end{array}$ & $\begin{array}{l}\text { Brotes ma) } \\
\text { manos, cc } \\
\text { sión limita }\end{array}$ & $\begin{array}{l}\text { res en hu- } \\
\text { transmi- }\end{array}$ & $\begin{array}{l}\text { Transmisión soste- } \\
\text { nida entre humanos }\end{array}$ \\
\hline \multirow[t]{3}{*}{$\begin{array}{l}\text { Fases } \\
\text { México }\end{array}$} & \multicolumn{7}{|c|}{$\begin{array}{c}\text { Prepandémica } \\
\text {-actividades de preparación- }\end{array}$} & Pandémica \\
\hline & & & Fases de & la etapa $p$ & indémica en & México & & \\
\hline & & Casos en $\mathrm{A}$ & $\begin{array}{r}\text { Casos en dos co } \\
\text { excepto Am }\end{array}$ & $\begin{array}{l}\text { ntinentes } \\
\text { érica }\end{array}$ & $\begin{array}{l}\text { Casos en } A \\
\text { casos er }\end{array}$ & $\begin{array}{l}\text { mérica, no } \\
\text { México }\end{array}$ & Casos en & México \\
\hline
\end{tabular}

Fuente: Plan Nacional de Preparación y Respuesta ante una Pandemia de Influenza. México, 2005

en aves y en humanos que permitan detectar oportunamente la presencia de los primeros casos en el país y disparar la alerta mundial a través de los mecanismos internacionales, si así se diera el caso.

Las líneas de acción del Plan contemplan prioritariamente: Coordinación, Vigilancia Epidemiológica, Atención médica, Difusión y movilización social, y Reserva estratégica.

\section{Coordinación}

A diferencia de otros problemas de salud pública, por su magnitud una pandemia de influenza rebasaría la capacidad y el ámbito de acción del sector salud. Es por ello que la coordinación entre instituciones de todos los niveles cobra una gran relevancia en la preparación de las acciones nacionales de contención.

La respuesta a una pandemia de influenza debe iniciar en el nivel local o municipal, con el apoyo de las autoridades estatales y federales. Para que ésta ocurra de manera fluida y adecuada, es necesario que los tres niveles de gobierno se encuentren coordinados de manera estrecha y se fortalezca la capacidad de respuesta en el ámbito de las unidades médicas y del personal del nivel operativo local

La Secretaría de Salud ha realizado talleres con la participación de autoridades sanitarias de las 32 entidades federativas que conforman geopolíticamente al país, incluyendo al personal del nivel estatal y jurisdiccional, quienes se encuentran actualmente en el proceso de desarrollo de sus respectivos planes de preparación y respuesta.
La coordinación entre instituciones, dentro y fuera del sector salud, es fundamental para la correcta implementación de las acciones de respuesta. Todas las instituciones y dependencias deberán conocer el papel que desempeñarán en el caso de una pandemia, con la finalidad de que la respuesta se establezca de manera ordenada y efectiva.

En el sector salud a nivel federal se está trabajando con las instituciones que proveen servicios de salud a la población para que desarrollen sus planes de preparación alineados con el Plan Nacional. En el nivel estatal, las secretarías de salud se encuentran en el proceso de incluir a las demás instituciones del sector en las acciones de respuesta. Lo mismo ocurre con el resto de instituciones de otros sectores relevantes.

Otro aspecto de la coordinación es el componente internacional, a través del cual se busca contar con información epidemiológica y sanitaria oportuna de otros países, y de avances científicos en materia de prevención y de control, así como obtener transferencia de tecnología y capacitación al personal.

En este sentido, México ha adquirido un papel de liderazgo en el ámbito mundial a través de su participación activa en los mecanismos multilaterales que actualmente trabajan para atender el problema de una eventual pandemia de influenza desde un punto de vista global.

México ha señalado de manera explícita la necesidad de que la comunidad internacional apoye a países que como el nuestro cuentan con la capacidad de convertirse en productores de antivirales y vacunas para hacer frente a la próxima pandemia. Igualmente, dada 
la amplia experiencia exitosa en la respuesta a emergencias sanitarias, México busca apoyar a la comunidad internacional, principalmente a los países de la región de América Latina en caso de que se presente esta eventualidad.

\section{Vigilancia epidemiológica}

La vigilancia epidemiológica es la piedra angular de la respuesta a una pandemia de influenza, pues es a través de ella que se podrán detectar de manera temprana los primeros casos de enfermedad que se presenten en el país y con ello disparar la alerta e iniciar las acciones de respuesta.

En México, el Sistema de Vigilancia Epidemiológica de Influenza ${ }^{11}$ está basado en una red de al menos 32 unidades centinela ubicadas en unidades de primer nivel en todo el territorio nacional; se encuentra en un proceso de fortalecimiento para mejorar la oportunidad y calidad de información que genera e incluye la detección de casos sospechosos de influenza (vigilancia sindromática de casos "influenza-like") y su confirmación mediante pruebas de laboratorio. Esta red servirá como base para la detección de casos y riesgos en caso de pandemia.

Actualmente se están incorporando nuevas tecnologías para la notificación inmediata de eventos epidemiológicos de interés, incluyendo casos sospechosos de influenza. Se dispone de una Unidad de Inteligencia Epidemiológica y Sanitaria (UIES) de nivel federal, que funge como centro nacional de notificación inmediata a través de telefonía y medios electrónicos (correo electrónico, fax, etc.); además, está equipada para realizar el monitoreo diario de medios masivos y otras fuentes de información nacionales e internacionales con las que se detecten situaciones de riesgo relevantes que pudieran reflejar la ocurrencia de eventos de interés epidmiológico y atención inmediata. En el marco de los convenios internacionales de intercambio de información epidemiológica, la UIES funciona también como punto de contacto permanente para los organismos multilaterales y las instituciones del extranjero.

En caso de que se presente una pandemia de influenza, la UIES también cuenta con la capacidad para realizar análisis epidemiológico rápido, que permita conocer con oportunidad el comportamiento de la enfermedad entre la población, a fin de alimentar la toma de decisiones en el país.

Con el propósito de mejorar la funcionalidad del sistema, se ha diseñado un plan de capacitación por etapas para el personal de salud (médicos, enfermeras, tra- bajadores sociales, personal de laboratorio, promotores, etc.), que incluye al que está en fase de formación en escuelas y facultades; se privilegian aspectos clínicos y epidemiológicos de la enfermedad, con lo que se busca mejorar el diagnóstico y la notificación.

También se dará capacitación al personal de áreas estratégicas que pueda en un momento dado apoyar en la detección de casos: de aduanas, migración, turismo, seguridad y otros sectores afines.

El laboratorio es otro aspecto fundamental de la vigilancia epidemiológica. A través de la capacidad diagnóstica es posible confirmar o descartar casos sospechosos, además de que permite la caracterización del virus para identificar si se trata del virus pandémico o cualquier otro virus de influenza que se encuentre circulando. En este caso, se busca fortalecer a los 32 laboratorios y coordinaciones que integran la Red Nacional de Laboratorios de Salud, a través de la incorporación de equipo y nuevas técnicas diagnósticas que permitan descentralizar la capacidad diagnóstica.

Debido a que se piensa que la próxima pandemia podría surgir de la mutación de un virus aviar, la Secretaría de Salud trabaja en coordinación con las autoridades de la Secretaría de Agricultura, Ganadería, Desarrollo Rural, Pesca y Alimentación (SAGARPA), la cual realiza acciones intensivas y permanentes de detección y control de la influenza aviar en todo el país. SAGARPA también lleva a cabo acciones de detección, caracterización de agentes y eliminación. Asimismo, cuenta con una campaña permanente de vacunación de aves para el control y eliminación de la enfermedad en México. ${ }^{12}$

\section{Atención médica}

En una pandemia de influenza, se rebasa rápidamente la capacidad de respuesta de los servicios de salud por el número de enfermos que demandan atención. El Plan contempla estrategias para expandir la capacidad de las instituciones de atención médica, tales como el uso de áreas habitualmente no empleadas en la atención (estacionamientos, áreas verdes, aulas), alta de pacientes que pueden ser atendidos en sus domicilios, suspensión de toda actividad no indispensable (cirugía electiva, procedimientos menores, etc.), entre otras. Estas modalidades innovadoras de "reconversión hospitalaria" se han desarrollado con la participación de expertos de la atención hospitalaria de nivel federal y estatal.

Asimismo se ha propuesto la aplicación de un esquema de triage para distribuir de manera racional a 
los pacientes entre las distintas unidades de atención de manera que ésta se proporcione de acuerdo con el nivel de gravedad (cuadro IV).

En caso de contar con una vacuna efectiva contra el virus asociado a una pandemia, se tiene contemplada una estrategia de distribución y aplicación de vacuna para la población.

México ha incorporado la vacunación contra influenza estacional en el esquema nacional de vacunación desde 2004. Esta vacuna se aplica en los meses de octubre a diciembre a niños de seis a 23 meses y a personas mayores de 65 años, además de otros grupos de edad con padecimientos cronico-degenerativos que los ponen a riesgo de complicaciones, en caso de contraer la enfermedad. Se ha considerado, asimismo, el incremento progresivo de la cobertura de vacunación antiinfluenza, lo que, además de proteger a la población contra la influenza estacional, disminuirá el riesgo de que al mismo tiempo, se encuentren el virus aviar y el virus humano en un individuo, y éste sirva como un "laboratorio natural" de intercambio genético que pudiera originar un nuevo virus, con la patogenicidad actual del virus de las aves y la capacidad de contagio entre humanos del virus humano, lo cual iniciaría una nueva pandemia.

Dentro del capítulo de atención médica, es necesario reforzar la capacitación al personal de salud en cuanto a medidas de bioseguridad. El personal que labora en unidades de atención es fundamental para la instrumentación de la respuesta y su protección resulta indispensable para evitar la exposición del recurso humano a riesgos innecesarios ante la presencia de influenza pandémica.

Finalmente, se ha conformado un grupo de expertos nacionales de los institutos nacionales de salud quienes están desarrollando, con base en la evidencia científica publicada y la experiencia clínica de nuestro país, guías de manejo y tratamiento de pacientes adultos y niños, en caso de una pandemia de influenza.

\section{Difusión y movilización social}

Las acciones de difusión y movilización social pretenden desarrollar mensajes y materiales para informar a la población en general sobre los riesgos que puede presentar una pandemia de influenza, y en el caso de que ésta se presente, de las acciones que pueden llevar a cabo para protegerse o para solicitar ayuda de parte de las autoridades sanitarias.

Para ello, se utiliza la estrategia de "vocero único" que ha resultado útil en México en casos de emergencias de salud pública, al permitir la emisión de mensajes unificados a la población. Esta estrategia se ha puesto en práctica en el nivel estatal en donde se cuenta ya con voceros únicos estatales que transmiten a sus poblaciones el mismo mensaje.

En este contexto, la estrategia de información a la población se desarrolla atendiendo a los principios de la promoción de la salud.

Una vertiente de esta línea de acción incluye el desarrollo de materiales para capacitación al personal de salud, así como a otro tipo de personal estra-

\section{Cuadro IV \\ EsQuema de tRIAGE PARA MANEJO DE PACIENTES EN UNA PANDEMIA DE INFLUENZA}

Tipo de paciente
Sano, sin sintomatología respiratoria

Referencia

Domicilio

Domicilio

Sintomatología respiratoria no compatible con influenza

Sintomatología sugestiva de influenza, sin complicaciones pulmonares y sin enfermedad crónica agregada

Cuadro clínico de influenza sin complicaciones pulmonares Centro de atención y aislamiento y/o enfermedad crónica controlada

Cuadro clínico de influenza con complicaciones pulmonares Unidad médica hospitalaria fija o de campaña y/o enfermedad crónica descompensada

Fallecido a consecuencia de un cuadro clínico sospechoso Servicio médico forense o confirmado de influenza

Fuente: Plan Nacional de Preparación y Respuesta ante una Pandemia de Influenza. México, 2005
Tratamiento Medidas de bioseguridad Sintomático y medidas de bioseguridad

Sintomático y medidas de bioseguridad Sintomático y medidas de bioseguridad Compensación de enfermedad crónica y complicaciones 
tégico en la respuesta a una pandemia de influenza, incluido el personal de aduanas, migración, seguridad pública, industria turística y protección civil, entre otro.

La estrategia de comunicación incluye material impreso preelaborado para su utilización en el momento en que se requiere, campaña de medios por fase de riesgo e información actualizada constantemente en la página electrónica de la Secretaría de Salud (www.salud. gob.mx).

\section{Reserva estratégica}

La correcta operación de la respuesta ante una pandemia de influenza requiere que las instancias de salud dispongan de los elementos e insumos necesarios para actuar de manera inmediata.

Para ello, se destinaron recursos específicos que permitieron la adquisición de una reserva de antivirales (oseltamivir y zanamivir), antibióticos de amplio espectro para el manejo de complicaciones, equipo de laboratorio, equipo para la protección del personal de salud, equipo de telecomunicaciones, además de otros insumos necesarios para la instrumentación de la respuesta.

El objetivo de la reserva es contar con los recursos para actuar de manera oportuna en donde se necesite; con la finalidad de contener el avance de la pandemia en el territorio nacional, se pretende que la reserva sirva de apoyo a la respuesta de los gobiernos de las entidades federativas y municipales, los cuales deberán también buscar el desarrollo de sus propias reservas.

La capacidad de producción de oseltamivir y zanamivir en el mundo es limitada, de ahí la importancia de hacer una reserva de dichos medicamentos para contar con ellos en el momento de la emergencia. Además, es de esperarse que el incremento en la demanda de ciertos productos condicione un alza desmedida de precios, por lo que se refuerza la relevancia de contar de manera anticipada con dicha reserva.

La oportunidad con la que se decidió que México efectuara la adquisición de una reserva de antivirales le permitió ser uno de los primeros países en el ámbito mundial en hacerlo y evitar así el retraso actual en la entrega del producto por las limitaciones de las farmacéuticas para enfrentar la gran demanda mundial.

Cabe aclarar que disponer de una reserva estratégica no significa simplemente tener un almacén de insumos, sino asegurar que todo aquello almacenado esté en buenas condiciones y considerar incluso el recambio oportuno de insumos con caducidad. Igualmente es necesario contar con todos los elementos necesarios para la distribución efectiva y eficiente de los insumos a los lugares en que se necesite durante una emergencia.

Finalmente cabe señalar que la reserva se ha concebido como un proceso de adquisición y abasto continuo que permitirá hacer frente a las necesidades de la población en materia de emergencias de salud pública. Se deberá considerar el presupuesto específico permanente para adquirir los elementos complementarios, además de ampliar el espectro de insumos que conformen la reserva.

\section{Investigación y desarrollo tecnológico}

Un elemento que se incluirá explícitamente en el Plan es el de investigación y desarrollo tecnológico, lo que permitirá disminuir la dependencia de nuestro país del mercado actual en materia de prevención, diagnóstico $\mathrm{y}$ tratamiento.

A pesar de que al no existir el virus pandémico es imposible contar con una vacuna para proteger a la población en caso de una pandemia, y conscientes de que la capacidad de producción de la vacuna para la influenza estacional es sumamente limitada en el plano mundial, las autoridades de salud federales de México han iniciado el complejo proceso junto con otros gobiernos y organismos multilaterales para que, a la larga, se llegue a la transferencia de tecnología y capacitación de personal que le confieran al país la capacidad de producción nacional, primero de la vacuna estacional y, eventualmente, de la pandémica. Con ello se busca que México sea autosuficiente en cuanto a la producción del biológico que se adquiere año con año para la vacunación contra influenza estacional, además de que se tendría la ventaja estratégica de contar con la vacuna necesaria para la población nacional en caso de una emergencia. Asimismo, se podrá producir biológico para apoyar a otros países de la región.

También será necesario buscar nuevas formas de diagnóstico y explorar alternativas de tratamiento, ya que sería deseable contar con pruebas diagnósticas rápidas que se apliquen en campo, y porque en la actualidad, como en el caso de la vacuna, la capacidad de producción de antivirales es insuficiente en el mundo, por lo que habrá que desarrollar nuevas opciones de producción o incluso de tratamiento.

\section{Conclusiones}

Ante los escenarios epidemiológicos actuales, es adecuado pensar en la inminencia de una nueva pandemia de influenza, pero la principal limitante consiste en que no se conoce en qué momento ocurrirá. Por ello, 
los gobiernos nacionales y locales, particularmente las autoridades sanitarias, deben redoblar esfuerzos en cuanto a las actividades de preparación.

Es complicado planear para un evento que se presume será catastrófico pero cuya ocurrencia es altamente impredecible. Sin embargo, no planear una respuesta acorde con la magnitud esperada del evento sería aun más catastrófico.

Antes de iniciar el proceso de planeación es necesario saber con qué presupuesto se cuenta, qué nivel de prioridad se asignará a las acciones de preparación, y en qué tipo de acciones se invertirá. De esa manera el proceso será más realista y tendrá mayores oportunidades de convertirse en acciones factibles de instrumentación.

El punto de partida de la planeación será el desarrollo de los escenarios nacionales, estatales o locales sobre los que se llevará a cabo todo el proceso, integrando a todas las instituciones relevantes desde este momento.

Uno de los principales obstáculos enfrentados en la elaboración del Plan Nacional de Preparación y Respuesta ante una Pandemia de Influenza es la resistencia de las autoridades de algunos sectores gubernamentales, así como de otros niveles organizativos. La tarea entonces ha sido mostrarles el impacto potencial que una pandemia tendría sobre el país y sus distintas regiones, y así convencerles de la importancia de trabajar en la planeación conjunta de la respuesta.

Un punto central de la planeación debe ser la consideración del riesgo al personal de salud, principal línea de defensa en una pandemia, así como las medidas necesarias para minimizar el impacto en este grupo. Actualmente hay importantes deficiencias en la aplicación de medidas de protección por parte del personal en la atención de pacientes, particularmente en el ámbito hospitalario. Por lo tanto, es fundamental trabajar en la sensibilización del personal y en la aplicación de estas medidas de manera cotidiana y no sólo para la contención de una pandemia de influenza. ${ }^{13}$

Contar con una vacuna antiinfluenza pandémica permitiría proteger a la población. Sin embargo, no se puede desarrollar un biológico contra un agente que aún no se conoce y con una capacidad de producción mundial limitada.

Si bien se encuentran múltiples centros de investigación científica tratando de desarrollar nuevas técnicas que permitan producir una vacuna efectiva y segura, lograrlo es aún lejano en un tiempo menor a los seis meses que como mínimo se requieren en la actualidad.

La estructura gubernamental y administrativa de cada país determinará la forma en que se organice la respuesta a una pandemia y, por lo tanto, dicha estructura deberá ser tomada en cuenta en la elaboración del plan.

Finalmente cabe destacar que los planes de preparación y respuesta son documentos dinámicos que constantemente requieren ajustes, adecuaciones y modificaciones para mantenerlos actualizados y útiles.

\section{Agradecimientos}

Los autores desean agradecer el valioso esfuerzo del personal del Centro Nacional de Vigilancia Epidemiológica y Control de Enfermedades (Programas Preventivos, Epidemiología e InDRE) y de las demás áreas de la Secretaría de Salud, así como de todas las dependencias que conforman el Comité Nacional para la Seguridad en Salud, que contribuyeron al desarrollo del Plan Nacional de Preparación y Respuesta ante una Pandemia de Influenza.

\section{Referencias}

I. Fock R, Bergmann H, Bussman H, Fell G, Finke E], Koch U et al. Influenza pandemic: Preparedness Planning in Germany. Euro Surveill 2002;7:1-5.

2. Paget WJ, Aguilera JF. Influenza pandemic planing in Europe. Euro Surveill 200I;6:136-40.

3. Bartlett JG, Hayden FG. Influenza A ( $\mathrm{H} 5 \mathrm{NI})$ :Will it be the next pandemic influenza? Are we ready? Ann Intern Med 2005; 143:460-462. 4. Shortridge KF. Pandemic influenza: a zoonosis? Semin Respir Infect 1992; 7:1 I-25.

5. Ferguson NM, Cummings DA, Cauchemez S, Fraser C, Riley S, Meeyai A et al. Strategies for containing an emerging influenza pandemic in Southeast Asia. Nature 2005;437:209-2/4.

6. De la Barrera CA, Reyes-Terán G. Influenza: Forecast for a pandemic. Arch Med Res 2005;36:628-636.

7. Organización Mundial de la Salud. Plan Mundial de la OMS de Preparación para una Pandemia de Influenza. Ginebra: Organización Mundial de la Salud, 2005.

8. Organización Mundial de la Salud.WHO Checklist for Influenza Pandemic Preparedness Planning. Ginebra: Organización Mundial de la Salud, 2005.

9. Secretaría de Salud.Acuerdo por el que se crea el Comité Nacional para la Seguridad en Salud. Diario Oficial de la Federación 2003 septiembre 22, México.

10. Secretaría de Salud. Plan Nacional de Preparación y Respuesta ante una Pandemia de Influenza. Ciudad de México: Secretaría de Salud, 2005. II. Secretaría de Salud. Sistema de Vigilancia Epidemiológica de Influenza. Ciudad de México: Secretaría de Salud, 1998.

12. Secretaría de Agricultura, Ganadería, Desarrollo Rural, Pesca y Alimentación. Ley Federal de Sanidad Animal. Diario Oficial de la Federación 1993 junio 18, México

13. Wilson $\mathrm{N}$ et al. The potential impact of the next influenza pandemic on a national primary care medical workforce. Hum Resour Health 2005;3:7. 